\title{
A New and Improved Method of Measuring the Pressure Dependence of Superconducting Transition Temperatures, $\mathrm{T}_{\mathbf{c}}(\mathrm{P})$
}

\author{
M. P. Raphael, M. E. Reeves", $\dagger$, E. F. Skelton ${ }^{\dagger}, *$, S. B. Qadri ${ }^{\dagger}$, C. Kendziora ${ }^{\dagger}$, and A. R. Drews $\ddagger$ \\ Department of Physics, Catholic University of America, Washington, DC 20064, USA \\ "Department of Physics, George Washington University, Washington, DC 20052, USA \\ ${ }^{\dagger}$ Condensed Matter and Radiation Sciences Division, U.S. Naval Research Laboratory, Washington, DC 20375-5320, USA

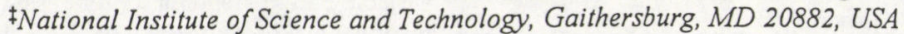

\begin{abstract}
A new technique for measuring $\mathrm{T}_{\mathrm{c}}(\mathrm{P})$ is reported. As the temperature of the DAC is reduced, instead of measuring the first harmonic of the ac susceptibility, $\chi_{1}$ ", the third harmonic, $\left|\chi_{3}\right|$, is measured. This results in a significant improvement in the minimum detection limit.

[superconductivity, transition temperatures]
\end{abstract}

\section{Introduction}

Conventional measurements have utilized ac susceptibility to determine the pressure dependence of the superconducting transition temperature, $\mathrm{T}_{\mathrm{c}}(\mathrm{P})$. Most groups use a primary coil coupled to two secondary coils: one containing the superconducting sample and the other empty for background subtraction $[1,2]$. Usually non-magnetic gasket materials with relatively large samples are used in order to separate the superconducting sample from the large, temperature dependent background of the gasket and the cell. This precludes reaching the highest possible pressures which are achieved by using high-strength gasket materials, such as Inconel and stainless steel, and small volumes of hydrostatic fluid. One group has solved this problem by measuring the

(a)

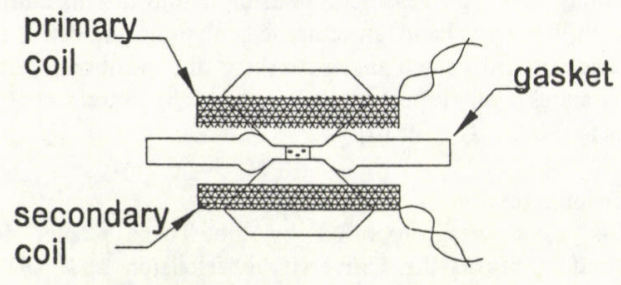

(b)

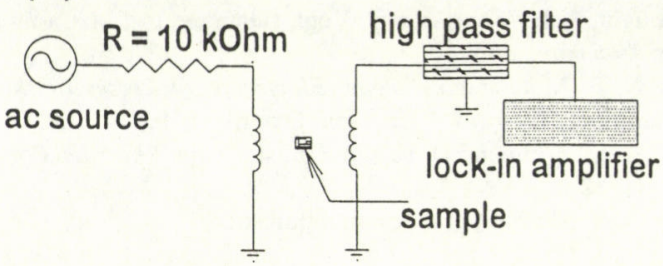

Figure 1. (a) the diamond anvil cell with primary and secondary coils (b) the circuit for measurement of the superconducting transition. second harmonic of the ac susceptibility [3]. Their technique relies on the use of a special non-magnetic stainless steel [4], which is not readily available and fairly complicated, custombuilt electronics, required to reduce the size of the background signal.

We will show in this paper that another approach, namely the measurement of the third harmonic of the ac susceptibility, can be used to determine $T_{c}(P)$ without the problems inherent in the above methods. Using readily available, off-the-shelf electronics and standard gasket materials (both magnetic and nonmagnetic), one can build a simple system to accurately measure $T_{c}(P)$ of small samples. Such a measurement sees only non-linear effects, such as the onset of flux motion in a superconducting sample and is not sensitive to the eddy currents produced in the gasket. Furthermore, in small magnetic fields, even magnetic gasket materials will exhibit no nonlinear effects. This technique can also be used to detect variations in $T_{c}$ that can arise from inhomogeneous samples and from pressure gradients in the hydrostatic medium.

The basic principle of the measurement is that the third harmonic of the ac susceptibility gives a measure of the losses induced by flux motion in a type- II superconductor. Above $T_{c}$, the only nonlinear effects arise from heating by eddy currents and are too small to detect. Well below $T_{c}$, the critical current $\left(j_{c}\right)$ is greater than the induced ac current in the sample Thus dissipation is small and a negligible third harmonic signal is generated. Close to $T_{c}$, however, $j_{c}$ drops to zero and hysteresis occurs in the presence of an alternating magnetic field. As predicted by Bean [5], the hysteresis generates a signal $\left(\mathrm{V}_{3 \mathrm{f}}\right)$ inversely proportional to $\mathrm{j}_{\mathrm{c}}$ at three times the fundamental frequency. Since $j_{c}$ goes to zero at $T_{c}$, a large peak is produced in sweeps of $\mathrm{V}_{3 \mathrm{f}} \mathrm{vs}$. $\mathrm{T}$, giving an extremely sensitive means of detecting the transition. As a result, the third harmonic of the ac susceptibility has been studied and used extensively for the characterization of type II superconductors [6-9].

\section{Experiment}

The diamond anvil cell setup is shown in Figure 1a. The bottom diamond is stationary; the top is moveable. Pressure 
is applied at room temperature by a screw which advances the upper diamond. The diamond culets are about $1 \mathrm{~mm}$ in diameter. Primary and secondary coils with several hundred turns each are wound from $25 \mu \mathrm{m}$ diameter wire and varnished onto the diamonds. Unless otherwise noted, the gaskets used here are made from hardened stainless steel. The gaskets are pre-indented and a $0.48 \mathrm{~mm}$ diameter hole is drilled in the center. The pressure medium used is silicone oil and the highest pressure achieved thus far with the cell is $8.4 \mathrm{GPa}$.

The gasket hole is loaded with small pieces of superconductor and ruby chips for in-situ pressure measurements. Care is taken to leave ample room for the pressure medium so that the diamonds do not press directly against the sample. As shown by Noack and Holzapfel, the shift in the R1 and R2 fluorescence peaks of the rubies, $\mathrm{d} \lambda / \mathrm{dP}$, is thermally invariant [10]. The value of $.365 \mathrm{~nm} / \mathrm{GPa}$ was used to determine the pressure [11]. By measuring the difference between fluorescence produced by rubies inside and outside of the gasket, the pressure can be determined within an uncertainty of $\pm 0.1 \mathrm{GPa}$ throughout the temperature range of our measurements. More specific details on the application and measurement of pressure in this particular cell are given elsewhere [1]

Figure $1 b$ shows the setup for measuring the superconducting transition. A lock-in amplifier [12] provides ac current to the primary coil. A high pass filter at the input of the lock-in is used to reduce the size of the fundamental voltage relative to that of the third harmonic. In our experiments only the amplitude of the third harmonic signal is recorded. The fundamental frequency used is $11.3 \mathrm{kHz}$ and the rms current supplied to the primary coil is $0.5 \mathrm{~mA}$. With this current, the ac field from the coil at the sample is
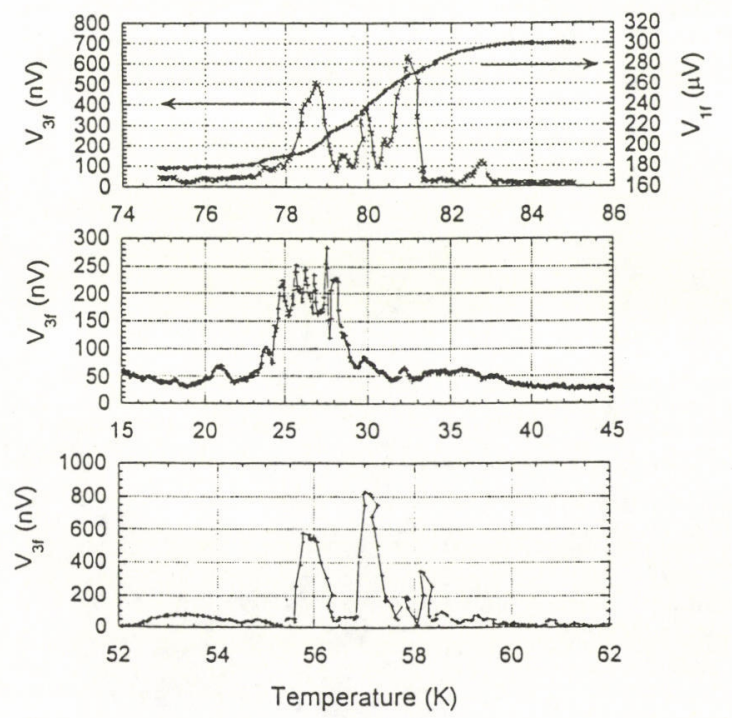

Figure 2. (a) Comparison of the first and third harmonic techniques on a Tl2201 single crystal, (b) $\mathrm{V}_{3 \mathrm{f}} \mathrm{vs}$. $\mathrm{T}$ for a T12201 crystal before annealing and (c) after annealing approximately 0.1 Gauss.

\section{Results and Discussion}

A. Studies of T12201 at $0.1 \mathrm{~Pa}$

Initial studies of inhomogeneities in superconductors and the effect of gasket materials on the third harmonic signal were carried out in a mock-up of the diamond anvil cell. Figure 2a shows the superconducting transition for a single crystal of $\mathrm{Tl}_{2} \mathrm{Ba}_{2} \mathrm{CuO}_{6+\delta}$. The data show a range of peaks, each corresponding to different inhomogeneous regions within the sample. The first harmonic voltage is plotted in the same graph, showing that with this technique, the inhomogeneities produced only a broadened transition. Figure $2 \mathrm{~b}$ shows a different T12201 crystal with a broad transition at $30 \mathrm{~K}$. Figure $2 \mathrm{c}$ shows the transition for the same sample after annealing at $400{ }^{\circ} \mathrm{C}$ for 16 hours in helium gas. The sharper transition at a higher $\mathrm{T}_{\mathrm{c}}$ indicates that the sample is now reduced and more homogeneous with respect to oxygen content. While this sample is much larger than those used in the diamond anvil cell, such features in the transition are even apparent in smaller gasket-hole-sized samples.

Figure 3 shows the transition of a $\mathrm{YBa}_{2} \mathrm{Cu}_{3} \mathrm{O}_{7+\delta}$ sample placed in the gasket hole of a $0.28 \mathrm{~mm}$ thick hardened Inconel gasket. Even though the gasket is magnetic, it produces no measurable effect on the background or the noise level of the signal. In conventional ac susceptibility, the signal from the magnetic gasket would dominate and the superconducting transition would be unobservable.

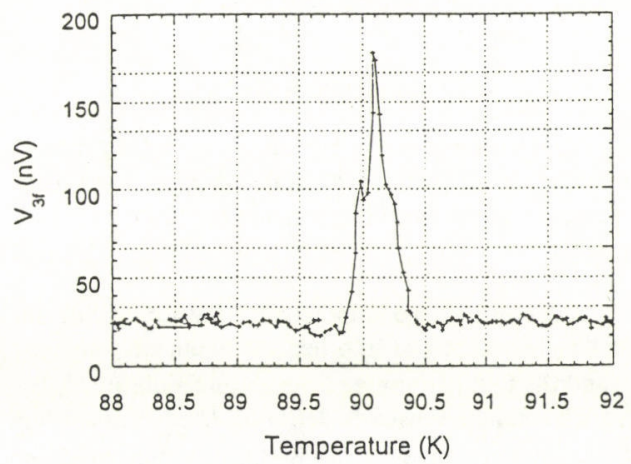

Figure 3. Transition of a YBCO single crystal in the presence of a hardened Inconel gasket. There is no detectable signal from the gasket.

\section{B. Studies of YBCO Under Pressure}

$\mathrm{T}_{\mathrm{C}}(\mathrm{P})$ data for $\mathrm{YBa}_{2} \mathrm{Cu}_{3} \mathrm{O}_{7+\delta}$, (YBCO) are shown in Figure 4. After each run, the diamond anvil cell is warmed to room temperature for 16 hours, the pressure is increased, and the cell is cooled again. At $0.1 \mathrm{~Pa}$ the sample displays a transition at $89.7 \pm 0.3 \mathrm{~K}$, where Tc is defined by the center position of the peak. The transition remains relatively sharp at $2.2 \mathrm{GPa}$ and $\mathrm{T}_{\mathrm{c}}$ increases to $93.1 \mathrm{~K}$. The increase in $\mathrm{T}_{\mathrm{c}}$ is consistent with earlier an earlier $\mathrm{T}_{c}(\mathrm{P})$ study on a YBCO 
sample with a similar $0.1 \mathrm{~Pa}$ transition temperature [13]. At $2.4 \mathrm{GPa}, \mathrm{T}_{c}(\mathrm{P})$ decreases to $91.8 \mathrm{~K}$ while the base of the peak broadens. At $2.6 \mathrm{GPa}$ the peak broadens further while separating into two distinct regions. When the pressure is increased to $3.6 \mathrm{GPa}$, peaks appear at $91.1 \mathrm{~K}, 91.9 \mathrm{~K}$ and $93.1 \mathrm{~K}$. Finally, the test at $3.6 \mathrm{GPa}$ was redone after warming the cell to room temperature for 16 hours. As seen in Fig. 4 , the $93.1 \mathrm{~K}$ peak has disappeared and a shoulder appears between 92.0 to $92.8 \mathrm{~K}$.

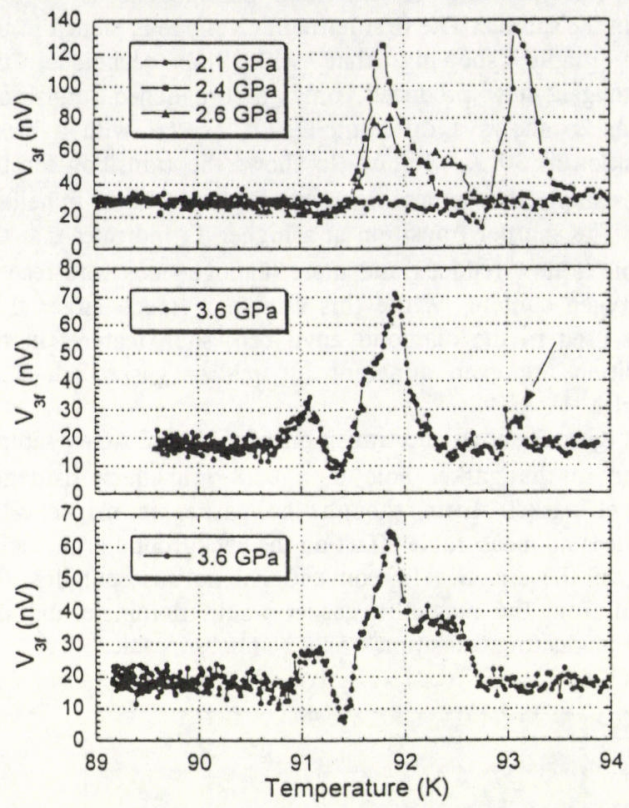

Figure 4. $\mathrm{T}_{c}(\mathrm{P})$ measurements on YBCO. The $3.6 \mathrm{GPa}$ shows 3 distinct peaks. Small changes in the peak structure are seen after warming the cell to room temperature for 16 hours (bottom panel).

The broadening of the superconducting transition in $T_{c}(P)$ tests has been observed in other measurements, such as resistivity and the first harmonic of the ac susceptibility $[1,2]$. However, such techniques usually define $T_{C}$ to be the onset or midpoint of the transition and the problem of the width is ignored. The above results show that such a definition of $T_{c}$ may not accurately represent what is really a set of distinct critical-temperature regions within the sample.

There are two possible explanations for the separation of $\mathrm{T}_{\mathrm{c}} \mathrm{s}$ described above. The first is that it is the result of an inhomogeneous pressure medium: there is observed broadening of the ruby fluorescence peaks for all applied pressures which indicates a nonuniform pressure within the gasket. Another explanation is that there is an uneven diffusion of oxygen upon application of pressure. We are studying this further with the use of different pressure media which will remain hydrostatic at higher pressures.

\section{Conclusions}

A technique of measuring $T_{c}(P)$ in the diamond anvil cell by the third harmonic of the ac susceptibility is described. It requires no background subtraction and allows the use of gasket materials made from hardened steels. In addition, the observed peak structure in the $\mathrm{V}_{3 \mathrm{f}}$ vs. $\mathrm{T}$ data allows separate regions of critical temperatures to be distinguished

\section{References}

[1] Charles C. Kim, M. E. Reeves, M. S. Osofsky, E. F. Skelton, D. H. Liebenberg, Rev. Sci. Instrum. 65, 992 (1994).

[2] J.S. Schilling, J. Diederichs, S. Klotz, R. Sieburger, it Susceptibility Studies of Superconducting Properties and Other Systems, edited by R.A. Hein, T.A. Francavilla, and D.H. Liebe (Plenum, New York, 1991) p. 107.

[3] Yu. A. Timofeev, Pribory I Technika Eksperimenta (in Russian) 5,. 190 (1992).

[4] V. V Struzhkin, private communication.

[5] C. P. Bean, Physical Review Letters 8, 250 (1962)

[6] R. Prozorov, A. Shaulov, Y. Wolfus, Y. Yeshurun, Physical Review B 52, 12541 (1995).

[7] S. Shatz, A. Shaulov, Y. Yeshurun, Physical Review B 48, 13871 (1993)

[8] T. Ishida, R. B. Goldfarb, Physical Review B 41, 8937 (1990).

[9] J. H. Claassen, M. E. Reeves, R. J. Soulen, Jr. , Rev. of Sci. Instrum. 62, 996 (1991)

[10] R. A. Noack and W. B. Holzapfel, in High Pressure Science and Technology, edited by K. D. Timmerhaus and M. S. Barber (Plenum, New York, 1979), Vol. 1, p.748.

[11] A. Jayaraman, Rev. Sci. Instrum. 57, 1013 (1986).

[12] Stanford Research Systems, model 830, Sunnyvale, CA 94089.

[13] H. A. Borges, R. Kwok, J. D. Thompson, G. L. Wells, J. L. Smith, Z. Fisk, and D. E. Peterson, Phys. Rev. B36, 2404 (1987). 